\title{
Editors' introduction Recent developments in the econometrics of structural change
}

\author{
Jean-Marie Dufour, Eric Ghysels \\ C.R.D.E. and Département de Sciences Economiques. Université de Montréal, Montréal. \\ Qué. H3C 3J7. Canada
}

Testing and analyzing structural change in econometric models is a very active research area. Up to a decade ago, econometricians mainly focused on linear regression models. In recent years, we have witnessed several new theoretical results for stationary and nonstationary dynamic models, nonlinear regression models, simultaneous equations and Euler equations models. This volume brings together a collection of papers which reflects the diversity of the recent developments on this subject. In October 1992, we had the pleasure of hosting at the Universite de Montréal a very stimulating C.R.D.E/Journal of Econometrics conference on Recent Developments in the Econometrics of Structural Change. We tried to assemble a program comprising the main topics on which important methodological advances have recently been made for the econometric analysis of structural change. The twelve articles which now appear in this volume are the result of this venture.

These papers cover four broad themes: (1) finite-sample tests of parameter constancy against the presence of structural change (Andrews, Lee, and Ploberger; Dufour and Kiviet); (2) asymptotic procedures for models with integrated time series (Ghysels and Perron; Gregory and Hansen); (3) asymptotic procedures for various dynamic models (Hamilton; Hidalgo and Robinson; Ploberger and Krämer); (4) Monte Carlo studies appraising the performance of recently developed tests (Campos, Ericsson, and Hendry; Diedold and Chen); and last but not least (5) empirical studies of structural change (Hackl and Westlund; Lütkepohl and Herwartz; Oliner, Rudebusch, and Sichel). The papers in the volume have been organized according to these themes and we shall now discuss them in this order.

The article by Andrews, Lee, and Ploberger considers a generalized changepoint problem which consists in testing the constancy of a standard normal linear regression model against an alternative where the regression may switch at $m$ unknown points $(m \geqslant 1)$. This problem is not regular because the breakpoints are not identified under the null hypothesis, so that standard 
distributional and optimality results for tests, both finite-sample and asymptotic, will not typically apply here. For dealing with it, the authors propose tests that maximize a weighted average power among tests of equal significance level, a principle which was suggested long ago by Wald (1942) but has almost never been exploited in this context or in econometrics in general (except for Andrews and Ploberger, 1994). For the case where the error variance is known, they derive a class of tests which are optimal according to this criterion, taking a Gaussian density with a single unspecified scale parameter $c$ as the weighting function. The distribution of the test statistic under the null hypothesis turns out to be free of nuisance parameters and can be established by simulation. The authors also argue that the power of the test is not too sensitive to the choice of $c$ and suggest that $c=\infty$ is a good practical choice. For the case where the disturbance variance is unknown (as it will typically be in practice), they suggest simply replacing the unknown variance by an estimate obtained under the alternative and show that the null distribution of the resulting statistic is also free of nuisance parameters, so its finite-sample distribution again can be established by simulation. In this case however, optimality in the sense of Wald is not demonstrated. Finally, the authors present Monte Carlo results where the size and power of the proposed tests are studied for models with two regressors and a one-time shift alternative ( $m=1)$. Among other things, the results indicate that the powers of the tests proposed are little influenced by the choice of $c$ and, in the unknown disturbance variance case, are close to being Wald-optimal. Their powers also compare favorably to those of other parameter constancy tests, such as CUSUM and sup $F$ tests.

In their contribution, Dufour and Kiviet study the problem of testing parameter constancy in linear dynamic models with one lagged dependent variable and an arbitrary vector of exogenous variables, again from a finite-sample perspective. Test procedures in such a context are typically based on asymptotic approximations whose reliability can be quite poor, and this is of course also the case for tests against the presence of structural change. The basic difficulty here comes from the fact that standard test statistics have null distributions which involve nuisance parameters. To obtain exact tests of parameter constancy for such models, the authors propose a two-stage strategy which consists in building first an exact confidence set for the coefficient $\lambda$ of the lagged dependent and then using the intimum and supremum of a 'static' parameter constancy test to obtain conservative and liberal critical regions. This yields a generalized bounds procedure (Dufour, 1990) with bounds that do not involve nuisance parameters. To obtain the exact confidence set for $\lambda$, the authors use extended regressor techniques proposed in Kiviet and Phillips (1992) and Dufour and Kiviet (1993). This general approach is then applied to obtain extensions to a dynamic model of various parameter constancy tests originally developed for static linear regressions, including: (1) analysis-of-covariance (Chow-type) tests; (2) CUSUM and CUSUM-of-squares tests; (3) predictive tests. For cases where 
the distribution of a test statistic cannot be computed analytically (e.g., CUSUM tests), they suggest replacing the original test by a Monte Carlo version of it which remains exact irrespective of the number of replications and becomes equivalent to the former as the number of replications increases. The authors also illustrate how their tests can be implemented by considering both artificial data and a dynamic trend model of gross private domestic investment in the U.S. It is of interest to note that the procedures proposed in this paper remain applicable even if the usual 'stationarity' assumption is not imposed (i.e., one may have $|\lambda| \geqslant 1)$. Further, it is clear that the approach used can be adapted to obtain dynamic extensions of other static regression procedures, such as the ones proposed in the first article of this volume (Andrews, Lee, and Ploberger).

The two next papers examine structural change problems in models with integrated time series. In the first one, Ghysels and Perron study the effect of filtering on time series with structural change. They observe that parametric models are most often fitted and tested for structural change from filtered data. A prime example is the one of seasonal adjustment. Such filters as well as others typically involve smoothing a time series and thus have a tendency to disguise structural instability. The paper analyzes both theoretically and by simulations the effect of linear filtering on the statistical properties of various tests in the presence of structural change. Three classes of tests are considered: namely, (1) tests for a unit root allowing for the presence of a change in the trend function, as discussed by Perron (1989), Banerjee, Lumsdaine, and Stock (1992), and Zivot and Andrews (1992), among others; (2) tests for changes in a polynomial trend function for a dynamic time series model, proposed by Gardner (1969), MacNeil (1978), and Perron (1991), and finally (3) tests for parameter instability with unknown change point for a general class of (nonlinear) models, discussed by Andrews (1993). For the first class of tests, the authors find that filtering data can have significant, undesirable effects on the power of tests, both asymptotically and in finite samples. On the other hand, no size distortions appear to occur. The situation is quite different for the second class of tests. Here, the authors show that filtering induces size distortions, even asymptotically. For an analytically tractable and simple case of a test proposed by MacNeil (1978), the authors obtain formulae characterizing the local size distortions and size-adjusted loss of power due to filtering. After an extensive Monte Carlo study, covering all three classes of tests, the paper concludes with an empirical application illustrating the effect of filtering in practice. Using seasonally adjusted and unadjusted series, the authors report several cases where the outcome of test statistics critically depends on the filtering of data.

On the same topic of structural change involving integrated processes, Gregory and Hansen propose extensions of tests for cointegration based on modifications of augmented Dickey-Fuller and Phillips (1987) type statistics. The null hypothesis is the usual hypothesis of no cointegration, but the authors consider an alternative where a cointegration vector may have shifted under the 
alternative. As the timing of the breakpoint is unknown, one can view the tests proposed by Gregory and Hansen as multivariate extensions of Banerjee, Lumsdaine, and Stock (1992), Perron and Vogelsang (1992), and Zivot and Andrews (1992). The authors provide a formal discussion of the Phillips-type tests $\left(Z_{t}\right.$ and $\left.Z_{\alpha}\right)$ with a characterization of their asymptotic distribution. Critical values are provided for up to four regressors in the statistical model. The augmented Dickey-Fuller statistic is discussed more informally. The finitesample behavior of the tests is investigated by Monte Carlo simulations, which indicate that the $Z_{t}$-type statistics appear to have the best size and power properties. They complement their paper with an empirical study of U.S. money demand using both annual and quarterly time series. Mixed evidence regarding the stability of long-run money demand is found. The authors also draw attention to the fact that their tests are complementary to recent work by Hansen (1992) and Quintos and Phillips (1993).

The paper written by Hamilton is the first of three which cover asymptotic procedures for analyzing structural change in various dynamic models. Hamilton (1989) proposed a Markov switching-regime model which has received wide attention in recent years. Here he proposes several diagnostic tests which are based on the Lagrange multiplier principle and the general approach to specification testing developed by Newey (1985), Tauchen (1985), and White (1987). At the center of the paper is an expression for the score function of a Markov switching model which permits calculation of tests for omitted autocorrelation, omitted ARCH, misspecification of the Markovian dynamics, and the absence of other explanatory variables. A Monte Carlo analysis of the proposed tests reveals that in small samples (i.e., 50 observations), severe size distortions may occur for all the Newey-Tauchen-White tests and LM tests for misspecification of the variance. In sample sizes of 100 observations, the author finds that the tests perform well. Finally, the paper reexamines the Engel and Ilamilton (1990) study of foreign exchange rates. None of the tests rejects the original specification, though for the British pound, the acceptance is borderline at $5 \%$. There is also evidence of a regime shift for both the French franc and the German mark, not captured by the models.

Hidalgo and Robinson study the problem of testing the constancy of regression coefficients in linear regressions with errors that follow a long-memory process. Such processes, which include various fractionally integrated processes, have autocorrelation structures which decay more slowly than those of stationary ARMA processes, while remaining stationary, and thus provide an interesting intermediate model between stationary and integrated ARMA processes. The article by Hidalgo and Robinson is really the first one on testing parameter constancy in such a context. For linear models with Gaussian disturbances and either nonstochastic regressors (e.g., polynomial time trends) or strictly exogenous regressors, the authors propose an analysis-of-covariance test against a one-time shift at a given break point. Since standard asymptotic 
methods are not applicable here, the main problems studied consist in finding the appropriate rate of convergence factor, which requires one to consistently estimate the 'decay' coefficient of the long-memory error process, and the asymptotic covariance matrix of the difference between two estimates of the regression coefficients based on two different subperiods. Finally the authors provide Monte Carlo results which suggest that their procedure is quite reliable and powerful in samples of sufficient size (say, greater than 100).

Ploberger and Krämer consider the problem of testing for the presence of structural change in lincar regressions with trending regressors of general form (deterministic or stochastic), which can be viewed as another non-standard situation. The main difficulty here is that the asymptotic null distributions of test statistics for parameter instability typically depend on the form of the trends. Finding the appropriate set of critical values in such a case may require a complicated process for establishing appropriate trend models. To avoid modeling trends, they propose instead a CUSUM-type test based on a sum of squared cumulative sums of OLS residuals, and show that the asymptotic null distribution of this statistic can be uniformly bounded over a wide class of trending regressors which include both polynomial trends and integrated processes. The asymptotic distribution of the CUSUM statistic under a class of local alternatives is also characterized. Finally, the authors present a small set of Monte Carlo results on the size and power of the proposed procedure.

The next two papers contain a wealth of results, based on large-scale Monte Carlo experiments, on the properties of various tests relevant to the analysis of structural change in time series models. Campos, Ericsson, and Hendry compare several tests of the hypothesis of no cointegration when the marginal process has a structural break. Note this form of structural change is different from a break in the cointegration equation studied by Gregory and Hansen. Campos et al. consider a relatively simple bivariate autoregressive process of order one (in error correction form) where the marginal process of one of the variables may have a shift in its drift coefficient. An important issue the authors focus on consists in comparing two-step Engle-Granger tests, using a Dickey-Fuller (DF) statistic, with single-step dynamic model procedures which test appropriate restrictions on the error-correction (ECM) representation. The authors find that the presence of a structural break in the marginal process has little effect on the size of the tests, but the ECM-based tests tend to be appreciably more powerful than the DF residual-based tests. This provides a strong argument for preferring the ECM-based procedures to the two-step procedures.

Diebold and Chen study the size properties of various structural stability tests against a one-time break at an unknown time, in the context of a simple AR(1) model. The main tests considered are the SupW, SupLM, and SupLR tests proposed by Andrews (1993), and bootstrapped versions of the same tests which are proposed by the authors. The main findings of the simulation are that the critical values based on the asymptotic distributions of the sup statistics 
proposed by Andrews lead to serious overrejections in finite samples while, on the contrary, the tests based on the bootstrap procedure lead to remarkably reliable tests. Clearly these results suggest that bootstrapping should be considered more carefully in this context.

The volume concludes with three empirical studies. The first one, by Hackl and Westlund, is an empirical study of international telecommunication demand which examines short- and long-term price elasticities for telecommunications between Sweden and three destination countries (Germany, the U.K., and the U.S.). The authors provide evidence from two approaches to stability analysis, Kalman filtering and moving local regressions, to reveal time variation in elasticities. For Germany and the U.K. they find gradual increases in price elasticity over a 14-year period, while for the U.S. they find a decrease. Empirical studies of telecommunication demand are important, as there is not a welldefined theoretical framework. Because empirical elasticity estimates are used in pricing policies, it is quite important to investigate their stability. The Hackl and Westlund study is therefore a timely study of structural change in this context.

Lütkepohl and Herwartz on the other hand consider the Flexible Least Squares (FLS) method, suggested by Kalaba and Testfatsion $(1989,1990)$, for recursively estimating coefficients of regression models which allow for time variation in the parameters. The FLS algorithm can capture smoothly evolving parameters as well as erratic coefficient changes. The latter case may include periodic parameter changes due to seasonality. The authors present a Kalman algorithm framework for FLS and apply it to quarterly West German income and consumption time series. The method is presented as a descriptive tool, the main advantage being its flexibility regarding the stochastic nature of the time varying parameters.

In the last paper, Oliner, Rudebusch, and Sichel assess the stability of empirical Euler equations for investment. They followed the suggestion of Ghysels and Hall (1990a, b) to use structural stability tests as a means to judge the adequacy of 'deep structural parameter' representations via a set of Euler equations estimated with the generalized method of moments estimator. Indeed, as emphasized in Dufour $(1982,1989)$, testing for structural change is an especially natural model diagnostic motivated by the Lucas econometric policy evaluation critique, and even more so in Euler equations whose very purpose is precisely to answer that critique. Moreover, the standard $J$-statistic used with such models is not suitable to diagnose structural change, because its asymptotic local power against alternatives characterized by parameter drift is zero (see Ghysels and Hall, 1990a). Oliner, Rudebusch, and Sichel examine an investment Euler equation which is typical of those previously encountered in the literature. They analyze stability by applying a set of tests involving pairwise comparisons of parameter estimates obtained from split samples. They also consider sequential tests for structural change. The Euler equations are based on a CobbDouglas production model with quadratic adjustment costs estimated with U.S. 
data over a 30-year sample of quarterly series. Their analysis reveals quite clearly that the Euler equation approach is not very successful in capturing 'deep structural' parameters, since a considerable degree of parameter instability is detected.

Before closing, we would like to take this opportunity to thank the editors of the Journal, in particular Cheng Hsiao, for their support, advice, and help. Financial support was provided by the Canadian Agency for International Development (PARADI program), the Centre de Recherche et Développement en Économique (C.R.D.E.), and the Départcment dc Scienccs Economiques of the Université de Montréal. We are deeply grateful to all the authors, discussants as well as the other conference participants for their input and contributions. Special thanks go to the referees of the papers, who devoted their time to the improvement of the quality of the contributions to this volume. Finally, the organizational skills of the staff of the C.R.D.E. were an invaluable asset which allowed the success of the conference: we would like to express our gratitude to Sharon Brewer-Moscato, Chantal Brunet, Francine Martel, Josée Vignola and, in particular, Marie-Christine Thirion.

\section{References}

Andrews, D.W.K., 1993, Tests for parameter instability and structural change with unknown change point, Econometrica 61, 821-856.

Andrews, D. and W. Ploberger, 1994, Optimal tests when a nuisance parameter is present only under the alternative, Econometrica 62, 1383-1414.

Banerjee, A., R.L. Lumsdaine, and J.H. Stock, 1992, Recursive and sequential tests of the unit-root and trend-break hypotheses: Theory and international evidence, Journal of Business and Economic Statistics 10, 271-288.

Dufour, J.-M., 1982, Recursive stability analysis of linear regression relationships: An exploratory methodology, Journal of Econometrics 19, 31-76.

Dufour, J.-M., 1989, Investment, taxation and cconometric policy cvaluation: Some cvidence on the Lucas critique, in: P. Hackl, ed., Statistical analysis and forecasting of economic structural change (Springer-Verlag, Berlin) 441-473.

Dufour, J.-M., 1990, Exact tests and confidence sets in linear regressions with autocorrelated errors, Econometrica 57, 335-355.

Dufour, J.-M. and J.F. Kiviet, 1993, Exact inference methods in first-order autoregressive distributed lag models, Discussion paper (C.R.D.E., Universite de Montréal, and Faculty of Economics and Econometrics, University of Amsterdam).

Gardner, L.A., 1969, On detecting changes in the mean of normal variates, Annals of Mathematical Statistics 40, 116-126.

Ghysels, F. and A. Hall, 1990a, Are consumption-based intertemporal capital asset pricing models structural?, Journal of Econometrics 45, 121-139.

Ghysels, E. and A. Hall, 1990b, A test for structural stability of Euler conditions parameters estimated via the generalized method of moments, International Economic Review 31, 355-364.

Hamilton, J.D., 1989, A new approach to the economic analysis of non-stationary time series and the business cycle, Econometrica 57, 357-384.

Hansen, B.E., 1992, Tests for parameter instability in regressions with I(1) processes, Journal of Business and Economic Statistics 10, 321-335. 
Kalaba, R. and L. Testfatsion, 1989, Time-varying linear regression via flexible least squares, Computers and Mathematics with Applications 17, 1215-1245.

Kalaba, R. and L. Testfatsion, 1990, Flexible least squares for approximately linear systems, IEEE Transactions on Systems, Man, and Cybernetics SMC-20, 978-989.

Kiviet, J.F. and G.D.A. Phillips, 1992, Exact similar tests for unit roots and cointegration, Oxford Bulletin of Economics and Statistics 54, 349-367.

MacNeil, I.B., 1978, Properties of sequences of partial sums of polynomial regression residuals with applications to tests for change of regression at unknown times, Annals of Statistics 6, 422-433.

Newey, W., 1985, Maximum likelihood specification testing and conditional moment tests, Econometrica 53, 1047-1070.

Perron, P., 1989, The great crash, the oil price shock and the unit root hypothesis, Econometrica 57, 1361-1401.

Perron, P., 1991, A test for changes in a polynomial trend function for a dynamic time series, Discussion paper (C.R.D.E., Université de Montréal, Montréal).

Perron, P. and T.J. Vogelsang, 1992, Nonstationarity and level shifts with an application to purchasing power parity, Journal of Business and Economic Statistics 10, 301-320.

Phillips, P.C.B., 1987, Time series regression with a unit root, Econometrica 55, 277-301.

Quintos, C. and P.C.B. Phillips, 1993, Parameter constancy in cointegrating regressions, in: J.-M. Dufour and B. Raj, eds., New developments in time series econometrics, Studies in empirical economics (Springer-Verlag, Heidelberg) 119-150.

Tauchen, G., 1985, Diagnostic testing and evaluation of maximum likelihood models, Journal of Econometrics 30, 415-443.

Wald., A., 1942, On the power function of the analysis of variance test, Annals of Mathematical Statistics 13, 434-439.

White, H., 1987, Specification testing in dynamic models, in: T.F. Bewley, ed., Advances in econometrics, Fifth world congress, Vol. I (Cambridge University Press, Cambridge) 1-58.

Zivot, E. and D.W.K. Andrews, 1992, Further evidence on the great crash, the oil price shock and the unit root hypothesis, Journal of Business and Economic Statistics 10, 251-270. 